\title{
La perception de l'ennemi dans les lettres des combattants français de la Grande Guerre
}

The Perception of the Enemy in French First World War Soldiers' Letters

\section{Sylvie Housiel}

\section{(2) OpenEdition \\ Journals}

Electronic version

URL: http://journals.openedition.org/aad/1744

DOI: 10.4000/aad. 1744

ISSN: 1565-8961

Publisher

Université de Tel-Aviv

\section{Electronic reference}

Sylvie Housiel, « La perception de l'ennemi dans les lettres des combattants français de la Grande Guerre », Argumentation et Analyse du Discours [Online], 13 | 2014, Online since 14 October 2014, connection on 21 December 2020. URL : http://journals.openedition.org/aad/1744 ; DOI : https:// doi.org/10.4000/aad. 1744

This text was automatically generated on 21 December 2020 .

\section{(c) (i) $\ominus$}

Argumentation \& analyse du discours est mis à disposition selon les termes de la licence Creative Commons Attribution - Pas d'Utilisation Commerciale - Pas de Modification 4.0 International. 


\title{
La perception de l'ennemi dans les lettres des combattants français de la Grande Guerre
}

The Perception of the Enemy in French First World War Soldiers' Letters

\author{
Sylvie Housiel
}

\section{Introduction}

Un siècle après la rédaction des lettres émanant du front de 1914 à 1918, l'intérêt porté à la parole des combattants français de la Grande Guerre ne faiblit pas. Antoine Prost et Jay Winter précisent que, parmi les sources exploitées, archives et documents deviennent des «dossiers de la guerre [qui] entrent dans le domaine public » (2004: 246), constituant ainsi des «lieux de mémoire que l'on conçoit comme des conservatoires des traces de ceux qui ont fait l'histoire " (ibid.). De leur côté, Rémy Cazals et Frédéric Rousseau reconnaissent la contribution et la particularité du témoignage des combattants pour une "histoire à dimension humaine »: «C'est précisément par ses innombrables apports singuliers d'expériences partagées par des millions d'individus que le témoignage apparaît alors comme la source la plus précieuse » $(2001: 51,52)$. C'est dans cette perspective que les lettres, «écrits rédigés dans l'instant », sont « des sources "directes" » qui présentent « l'avantage d'offrir un certain nombre de conclusions concordantes permettant de dresser un tableau assez fiable du soldat français de 1914-1918, et en particulier des représentations qui ont orienté ses manières de penser et d'agir entre ces deux dates " (Audoin-Rouzeau \& Becker 1995 : 307).

Cet article explore les modalités selon lesquelles l'ennemi a été perçu dans un échantillon de lettres rédigées par des combattants français sur le front, et sur celui de Verdun plus particulièrement, en 1916. A l'aide des instruments de la linguistique de l'énonciation, de l'analyse du discours et de l'argumentation, il cherche à dégager ce qui se construit dans les échanges avec un « autre » affectivement proche. On part donc 
de l'hypothèse qu'une analyse attentive et serrée des lettres, de leur dynamique et de leur logique propres, peut compléter le savoir considérable déjà accumulé sur la question du rapport à l'ennemi. Il s'agit ainsi de comprendre, à travers une analyse ponctuelle d'éléments langagiers mais aussi culturels et historiques, comment se construit une image nouvelle et inattendue de l'Allemand, produite par l'identification dans une souffrance et un destin communs. En s'identifiant au soldat allemand dans les épreuves qu'il endure, en présentant les deux armées comme logées à la même enseigne, les discours des combattants français retravaillent et déplacent les représentations initialement charriées par les médias et les discours officiels. Ils justifient en même temps, pour les combattants des deux camps, les très controversées «trêves" des combats et « rapprochements » des armées en présence, sur lesquels il conviendra de revenir. En même temps, on entend montrer comment les épistoliers ont cherché à justifier leurs nouvelles perceptions, leurs points de vue et leurs émotions personnelles sur leur vécu des tranchées, afin d'obtenir l'adhésion de leurs destinataires.

Les épistoliers ont été choisis en raison de leur diversité dans l'âge, le statut social, l'unité, le secteur de combat et les fonctions militaires. Raoul Bouchet est un jeune artilleur, mobilisé, d'origine rurale ; Jean Pottecher est étudiant en médecine, pacifiste, socialiste, infirmier et volontaire sur le front; le commandant Henri Bénard est un officier de carrière à la retraite, d'un certain âge (58 ans) et volontaire au combat. Notons que Pottecher est issu d'un milieu social différent de celui des deux autres épistoliers, et que son statut de pacifiste conditionne sans doute la manière dont il élabore l'image de l'adversaire. Cette différence, ainsi que celle qui sépare un jeune rural appelé sous les drapeaux d'un militaire de carrière d'âge mûr qui s'est porté volontaire, est voulue : elle permet d'éclairer ce qui réunit des combattants si divers dans leur nouveau rapport à l'ennemi.

En ce qui concerne le choix des secteurs et surtout celui de Verdun, il se justifie par le fait qu'il est de ceux où on ne s'attend guère au genre de phénomène qu'on tente de mettre en évidence ici. Considéré comme l'une des zones de grande bataille, il ne fait pas partie de ce que l'on nomme généralement des secteurs "tranquilles». Dans un secteur particulièrement "agité", on a voulu se concentrer sur des lettres qui n'abordent pas explicitement et longuement le thème en question, comme l'ouvrage de Barthas (2003 [1977]) par exemple (précisons en passant qu'il s'agit d'un carnet de guerre et non d'une correspondance), mais bien plutôt laissent percer un changement d'attitude dans des échanges ordinaires qui s'effectuent dans des circonstances extraordinaires.

Une dernière remarque: lorsqu'on se propose d'éclairer la question du rapport des combattants français à l'ennemi ${ }^{1}$, il convient en un premier temps de rappeler qu'elle se situe au sein d'une polémique qui a opposé deux courants, dans le champ de l'histoire culturelle. Le premier, basé sur la thèse d'une certaine "acceptation » du combat, pose que les soldats français ont accepté la prolongation du conflit pendant quatre ans et demi en raison d'une «culture de guerre » définie comme " un corpus de représentations du conflit cristallisé en un véritable système donnant à la guerre sa signification profonde » (Audoin-Rouzeau \& Becker 2000 : 145). L'autre courant objecte que les soldats ont combattu par obligation, par devoir, par soumission, ou encore par résignation sans "suspecter [toutefois] le patriotisme des combattants de 14-18 " (Rousseau \& Cazals 2001: 142), mais non par «acceptation». On perçoit les enjeux idéologiques et sociaux de cette polémique d'historiens qui porte sur un sujet toujours 
brûlant : le rapport du combattant à la violence de guerre, et la capacité du patriotisme à motiver les hommes appelés à se battre. On comprend dans ce contexte que le rapport des combattants à l'ennemi et la notion de «fraternisation ${ }^{2}$ " puissent être un sujet de controverse. Mon propos n'est pas de prendre parti dans la polémique, mais de vérifier à travers une analyse approfondie des lettres la façon dont s'y construit, par un changement de vocabulaire pour qualifier ou désigner l'ennemi, par la mise en place de comparaisons entre les soldats des deux camps, par le maniement de l'implicite, un rapport nouveau aux Allemands qui n'est plus simplement «l'ennemi à abattre». On pourra ainsi voir, en fonction de ce qui ressort des échanges épistolaires, ce qu'on peut entendre par le terme tant discuté de « fraternisation ».

\section{Le contexte historique de l'écriture}

Il convient tout d'abord de situer l'épistolier et son discours dans son contexte historique, au début de cette année 1916 : pour explorer les textes, «il faut prendre en compte différentes couches de données: il faut interroger l'archive» (Amossy 2000 : 97). L'année 1915 qui est celle de l'installation de la guerre des positions, a été marquée par une violence extrême (Horne 2010). Elle se termine, du côté des Alliés, par des échecs dus essentiellement à des divergences de vues entre les gouvernements ${ }^{3}$ et au manque de coordination entre les efforts des armées. À l'entrée de l'année 1916, l'Empereur allemand, Guillaume II, choisit d'attaquer sur le front Ouest. Même si le Commandement français n'est pas surpris stratégiquement, le secteur de Verdun, longtemps négligé, possède des organisations défensives incomplètes : «En 1915, les Français avaient commencé à traiter [le secteur de Verdun] en zone calme et à dépouiller les forts de leur artillerie, ignorant les renseignements qui évoquaient une attaque ennemie en préparation " (Strachan 2005 [2003]:182). La première phase des offensives se concrétise par l'attaque brusquée du 21 février 1916 (Gazin \& Segonne 1931 : 54-59) par les Allemands qui s'emparent du fort de Douaumont. En l'espace de huit semaines (du 21 février au 20 avril 1916), ils tirèrent plus de huit millions d'obus et, durant les trois cents jours de la bataille de Verdun (du 21 février au 15 décembre 1916), il fut tiré, toutes armées confondues, soixante millions d'obus de tous calibres (Becker 2004 : 43). Et, « quand le silence retomba, en décembre, les pertes allemandes s'élevaient à 337000 hommes, dont 143000 tués, et les françaises à 377231 hommes, dont 162440 tués » (Strachan 2005 : 183).

Le bilan est lourd et certains secteurs sont loin d'être "tranquilles ». Ce sont ceux-là qui intéressent. En effet, malgré leurs horribles conditions, les combattants continuent à écrire. Plus étonnant, certains vont même jusqu'à effectuer une identification avec l'ennemi dans les épreuves qu'ils endurent. Commençons par examiner quelques échanges de Bouchet, puis ceux du commandant Bénard et enfin d'autres émanant de Pottecher. Chaque épistolier, ainsi que la correspondance dont émergent les extraits, sera présenté plus en détail au seuil des analyses.

\section{Mêmes tranchées, même punition...}

Les premières analyses portent sur la correspondance d'un jeune rural de 20 ans, Raoul Bouchet qui se trouve dans le secteur "... à droite sur le plateau de L... vers le bois X " (Bouchet 2002: 219). Agriculteur engagé volontaire en mai 1914 dans la cavalerie, 
mobilisé comme $2^{\mathrm{e}}$ canonnier conducteur d'un 75 au $20^{\mathrm{e}} \mathrm{RA}$ de l'A.D 17 , il est tué le 9.10.1916 au nord de Péronne (Somme). Sa correspondance qui débute le 7 mai 1914, couvre les trois années de guerre de l'épistolier qui se termine avec son décès en 1916 et comprend 326 missives.

Dans une lettre destinée à ses parents, il relate une scène quotidienne qu'il veut rassurante à leur égard : «le secteur n'est pas du tout meurtrier », et qu'il valide par son exposition personnelle - «moi-même, je suis parti des premières lignes à découvert »:

Sommes légèrement à droite sur le plateau de L... Le pays est superbe mais les tranchées sont d'une saleté effrayante, dans des endroits, il n'est pas rare de trouver $50 \mathrm{~cm}$ de boue, il y a même des endroits où des fantassins se sont enlisés. Naturellement, chez les Boches c'est la même chose, alors il n'est pas rare de voir des Français et des Boches sortir à découvert des premières lignes ; il n'est pas tiré un coup de fusil, le secteur n'est pas du tout meurtrier. Moi-même, je suis parti des premières lignes à découvert.

Quelques détails curieux : vers le bois X, les Boches et les Français ramassent du bois au même endroit et en même temps. Ce matin, un sous/officier boche est venu dans la tranchée occupée par le 90ème ; après avoir salué militairement, il a dit que le régiment dont il faisait partie, était puni de 60 jours de tranchée et qu'il arrivait de Russie. Les hommes sont mécontents et ont juré de ne pas tirer un coup de fusil, je vous assure que c'est drôle de voir ce calme. De notre côté, un caporal du 90ème a été leur rendre visite, il faut le voir pour le croire, tellement ça paraît invraisemblable (Bouchet 2002 : 219, 220 ; lettre du 13 janvier 1916 à ses parents).

$\mathrm{Au}$ premier abord, le premier paragraphe semble servir à exposer aux parents du jeune soldat des conditions de combat difficiles; le second offre une réflexion personnelle où il exprime son étonnement et le partage avec les siens. On verra toutefois comment se construisent des significations qui dépassent la littéralité de l'énoncé, et permettent de cerner des informations précieuses au-delà de ce qui se dit à la surface du texte.

Tout d'abord, le paradoxe entre le respect ou la crainte de la censure par l'épistolier qui, volontairement, ne cite pas l'endroit géographique où il se trouve «sur le plateau de L... ", " vers le bois X... », et la scène qui relate une trêve des combats entre les deux camps marque que le jeune combattant n'est pas conscient de la gravité des évènements qu'il rapporte. Pour lui, l'entente tacite du cessez-le-feu est une conséquence "naturelle» et évidente de leurs difficiles conditions communes de tranchées : "Naturellement, chez les Boches c'est la même chose, alors... ». La valeur argumentative de l'énonciation se situe au niveau du connecteur « alors » qui introduit « il n'est pas rare de voir des Français et des Boches sortir à découvert des premières lignes » et présente ce deuxième énoncé comme une conséquence directe du premier. La liaison ainsi établie entre la boue et la trêve permet de transférer sur la conclusion l'adhésion accordée aux prémisses (Perelman 2002 : 74). En d'autres termes, elle justifie la trêve établie entre les soldats des armées ennemies. La stratégie discursive à visée argumentative consiste ainsi à présenter combattants français et allemands " enlisés » dans des tranchées "d'une saleté effrayante", et à construire en même temps un groupe de combattants unis dans une commune "galère » et amenés à adopter des comportements similaires.

Toutefois, si le mécontentement des soldats allemands est explicitement exprimé :« Les hommes sont mécontents et ont juré de ne pas tirer un coup de fusil », celui des soldats français ne l'est qu'implicitement. Notons tout d'abord le statut accordé à l'affectation aux tranchées mais aussi et surtout au nombre de jours infligés : 60 . De plus, si les deux 
données sont clairement présentées comme une "punition", (non seulement les tranchées, mais en plus 60 jours!), c'est par rapport aux Allemands dont l'épistolier rapporte les propos : « il a dit que le régiment dont il faisait partie était puni de 60 jours de tranchée et qu'il arrivait de Russie ». Or, Bouchet avait déjà introduit une nette comparaison entre les tranchées allemandes et françaises : "Naturellement chez les Boches, c'est la même chose ». Le destinataire peut ainsi dégager ce que le discours se contente de sous-entendre sur la "punition » que représentent les tranchées pour les Français. Il en va de même de la décision de ne pas tirer. Dans "Les hommes sont mécontents et ont juré de ne pas tirer un coup de fusil », le et de coordination revêt le sens de "en conséquence", présentant la décision allemande de ne pas combattre comme une protestation collective. Celle-ci rejaillit indirectement sur les Français dans la mesure où elle se rattache à la formule impersonnelle qui précède : « il n'est pas tiré un coup de fusil », qui se rapporte aux deux camps.

La conclusion qu'il laisse supposer à ses destinataires par recoupements lui permet ainsi de dénoncer le sort réservé aux troupes dont il fait partie et le comportement qu'elles choisissent d'adopter sans se compromettre ni perdre la face (Goffman 1973). La nouvelle représentation de l'ennemi sur lequel « on » ne tire plus, et qui remet en cause le rôle de l'épistolier, celui de ses camarades et une structure sociale entière, renvoie en effet à la rupture dont parle Goffman, et aux risques qu'elle fait courir :

[...] l'acteur est souvent profondément impliqué dans un rôle, une organisation, et un groupe déterminé auxquels il s'identifie; il se perçoit lui-même comme quelqu'un à qui l'on peut faire confiance, qui ne provoque pas de rupture dans l'interaction ou qui ne déçoit pas les ensembles sociaux qui comptent sur la réussite de cette interaction. C'est pourquoi, lorsqu'une rupture se produit, il arrive qu'elle détruise l'image de soi autour de laquelle la personnalité de l'acteur s'est édifiée. [...] Les ruptures de représentation ont donc des conséquences à trois niveaux différents : celui de la personnalité, celui de l'interaction, et celui de la structure sociale. [...] (ibid. : 230).

À la fois tributaire de la censure militaire mais aussi de l'autocensure qu'il s'impose, on comprend plus aisément l'embarras de Bouchet à présenter à ses parents la "trêve " comme initiée par les Français : elle serait seulement «suivie » et explicable par des conditions de combat communes et lamentables. Elle introduit en effet une situation tout à fait nouvelle qui contredit une doxa préalable et commune aux interlocuteurs. Notons que l'épistolier présente lui-même la situation comme « invraisemblable » : « il faut le voir pour le croire ». Cette mise en mots permet de saisir la prise de position du locuteur qui s'étonne de la scène : «je vous assure que c'est drôle de voir ce calme ", mais ne la condamne pas. La stratégie utilisée autorise ainsi une introspection dans la nouvelle perception du combattant sur les événements en général et sur l'ennemi en particulier, dont il veut rendre compte à ses destinataires tout en cherchant à l'expliquer, voire la justifier.

La comparaison introduite par "c'est la même chose" qui se définit par le rapprochement, dans un énoncé, de termes ou de notions au moyen de liens explicites, a ici pour but principal d'éclairer, à l'intention des destinataires, un propos qui n'est pas toujours facile à appréhender: la "trêve" avec l'ennemi. De plus, la censure militaire est, rappelons-le, un autre facteur à prendre en compte. Ainsi, du sousentendu habilement mis en place par Raoul - «mêmes tranchées, même punition » - il est aisé de saisir à quelle conclusion l'épistolier veut amener ses destinataires: le mécontentement des deux armées adverses devant un piètre traitement identique justifie ce que Bouchet nommera quelques lettres plus loin «l'entente cordiale » (2002: 


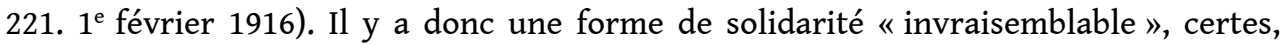
mais humaine et bien réelle par-delà les différences identitaires.

Le discours épistolaire exploite ici les ressources du sous-entendu, qui se distingue en ce qu'il « englobe toutes les informations qui sont susceptibles d'être véhiculées par un énoncé donné mais dont l'actualisation reste tributaire de certaines particularités du contexte énonciatif » (Kerbrat-Orecchioni 1986: 39). L'avantage du procédé est qu'il permet à Bouchet d'appuyer son argumentation sur le contenu de l'information en se gardant de porter une condamnation directe ou de plaider en faveur d'un comportement fraternel interdit, laissant aux destinataires la responsabilité de la conclusion : «Pour dire quelque chose, on fait dire par autrui qu'on l'a dit » (ibid. : 45, 46). En l'occurrence, on l'a vu, ce sont les Allemands qui «parlent» de punition de tranchées, et de refus de tirer, et non l'épistolier.

La scène est présentée par le biais du discours rapporté que l'épistolier relate avec l'autorité du témoin qui a entendu le propos. Il prend soin de se retrancher derrière un tiers - procédé qui «est bien souvent une manière habile parce qu'indirecte » de suggérer ce que l'on pense sans avoir à s'en porter garant (Kerbrat-Orecchioni 1978 : $60,61)$, en prenant ses distances par rapport à son énoncé. Qui plus est, rapporter, et, dans ce cas, valider la scène, présente un certain degré d'adhésion de la part de l'épistolier au fait rapporté. Même s'il cherche à se détacher des propos qu'il rapporte en les attribuant aux Allemands, le fait que l'énoncé soit livré aux destinataires montre que les faits rapportés sont jugés dignes d'intérêt. En d'autres termes, Bouchet ne prend pas la responsabilité du «dit», mais celle du "dire " lui est entièrement imputable.

\section{Les kamarades d'en face}

L'identification se fait beaucoup plus explicitement dès que la sphère de l'échange se situe de nouveau entre deux combattants :

Marie [sa sœur] m'écrivait, hier au soir, que l'on parlait sérieusement de la paix à Châtellerault [sa ville natale], moi, je n'y crois pas encore. Il est certain que nos voisins d'en face en sont fatigués et si ce n'était leurs pièces lourdes, les fantassins boches auraient vite fait, tous, kamarades (Ibid : 273 ; lettre à son beau-frère sur le front du 10 mai 1916).

On ne peut manquer de relever ici la transcription du terme kamarade directement emprunté à la langue allemande par l'emprunt graphique du k. Ce point est d'autant plus intéressant qu'il permet de supposer que l'épistolier a vu ce terme écrit pour pouvoir le reproduire exactement, vu son statut de jeune rural. De plus, Raoul pose comme une certitude (certain) l'arrêt des combats de la part des fantassins allemands (tous) si la décision leur appartenait. Cette assertion, lourde d'implicite, laisse entendre que l'épistolier connaît suffisamment bien les combattants allemands pour pouvoir rapporter leurs propres paroles. La reprise de la parole de l'Autre renforce non seulement la thèse d'une certaine " trêve ", mais soulève aussi indirectement celle d'un combat forcé, attribuable aux deux armées, en tout cas, à cette époque précise, en mai 1916, et dans ce lieu, à Verdun. 


\section{Un « malheureux Boche »}

La suite de la correspondance marque toujours une certaine résignation dans l'absurdité des combats que les deux camps subissent: "Il fait un temps très beau aujourd'hui, je suis au poste d'observation attendant qu'un malheureux Boche se montre pour l'envoyer dans le royaume du diable » (ibid. : 286 ; lettre à ses parents du 21 juin 1916). L'emploi du prédicat «malheureux » qui fait allusion dans ce contexte à la malchance, à l'aléatoire et à l'arbitraire ${ }^{4}$, déplace les propriétés que la propagande attribue à l'ennemi. La juxtaposition des deux termes - «malheureux boche » - déplace la valeur péjorative et stéréotypée du deuxième terme pour lui conférer une signification nouvelle (Jakobson [1963] 2003 : 49). Rappelons qu'à l'origine, l'appellation «boche " n'avait rien de commun avec les Allemands quand elle fait son apparition vers 1860 ; elle était alors un parisianisme au sens de mauvais sujet dans "l'argot des petites dames " (Delvau [1866] 1883), une abréviation parisienne de "caboche » qui signifiait "tête dure» (Sainean [1915] 1973 :10). Même pendant la guerre de 1870, le terme «boches » ne désignait en rien les Allemands, nommés alors les Prussiens. Ce n'est qu'après la guerre de 1870-71, que l'appellation se réfère particulièrement aux Allemands. De plus en plus courante dans le langage populaire, elle est reprise dans les casernes, les tranchées et dans la presse de la Grande Guerre.

Mais la guerre a de nouveau un impact sur le langage qui se renouvelle et se modifie sans cesse, laissant percevoir de nouvelles représentations. Ainsi le "barbare " et « l'ennemi à tuer » du discours médiatique de l'entrée en guerre ${ }^{5}$ se transforme en un « kamarade ", un " malheureux boche » - on y ajoutera " chez eux » - ce qui remodule entièrement la perception du soldat allemand. Ce procédé permet à Bouchet, d'une part, de dénoncer définitivement et clairement l'absurdité de la guerre et d'autre part, d'élaborer une nouvelle image du combattant allemand qui n'est plus conforme au stéréotype du soldat redoutable devenu désormais vulnérable. Le poilu ne nourrit pas de haine contre l'Allemand qu'il présente comme affaibli, et n'a pas non plus une haine systématique à son égard ${ }^{6}$ mais plutôt une certaine sympathie. Cette analyse montre comment l'image discursive de l'ennemi est entièrement reconstruite et modifie la représentation initiale diffusée par la propagande, ou, plus exactement, contribue à la détruire.

\section{Le visage humain de l'ennemi}

Si l'on pouvait penser que le genre de scènes vues jusqu'à présent se réfère uniquement à des jeunes combattants, qui plus est des ruraux, il n'en est rien. En effet, dans l'échange que le commandant Henri Bénard, 58 ans, officier d'active retraité, volontaire au combat, entretient avec son épouse, se construit la même image de «l'ennemi ». L'épistolier est dans le secteur des Éparges ${ }^{7}$ avec le $366^{\mathrm{e}}$. Il sera tué au combat le 28 février 1916. Sa correspondance qui débute le 4 août 1914 et couvre ses trois années de guerre jusqu'à son décès en 1916, comporte 336 lettres. Le rapport de compréhension, d'humanité, voire de camaraderie entre les soldats des deux armées que l'échange cidessous laisse percer, présente une ressemblance avec ce que mettent en avant les lettres de Bouchet, si ce n'est que le style et la forme en sont différents :

Aujourd'hui, avons eu des Boches qui sont venus se faire tuer dans nos fils de fer, un gros sous-officier nommé Kuhn et un jeune Prussien étudiant. Les deux sont morts 
et venons de les enterrer. Le jeune étudiant a vécu quelques heures. Il parlait bien le français. [...] Je suis très fier d'avoir une petite fille qui travaille si bien et décroche la première place en littérature. Et l'Allemand? Maman te donne-t-elle des devoirs? Tu sais qu'il en faut pour l'examen du brevet. Je crois plus que jamais que la guerre ne durera pas très longtemps et je pense toujours à notre voyage en Allemagne. Que ce sera bon d'être sorti de cette vie que menons! (Bénard 1999:

233. Lettre du 8 février 1916)

Le statut d'ancien militaire de carrière de Bénard ne l'autorise pas à présenter les faits à sa destinataire (sa fille), de la même façon, littéralement parlant, que Bouchet. De même, le statut des destinataires respectifs est à prendre en compte. Bouchet s'adresse à ses parents, à son beau-frère et à sa sœur, tous de la même origine sociale que lui. Bénard, quant à lui, s'adresse à sa jeune fille, encore à l'âge de la scolarité, et d'une façon indirecte, à son épouse d'origine alsacienne. Pour toutes ces raisons, mais surtout pour la dernière, il prend soin de présenter la « rencontre » entre les deux armées dans un décor de guerre: "sont venus se faire tuer dans nos fils de fer». Toutefois, on notera deux dimensions intéressantes dans la mise en mots; la première est l'absence d'hostilités ou d'offensive de la part des Français vis-à-vis des Allemands présentés comme prenant l'initiative de l'offensive ("ils sont venus »), et la deuxième dimension est la mort des Allemands, due à des fils de fer et non à des armes françaises. Dans cette première image, se dessine une certaine absurdité dans les circonstances de leur mort et implicitement dans celles de la guerre.

Ensuite, attribuer des prédicats aux soldats allemands, pour les décrire physiquement ("gros sous-officier») et socialement ("jeune Prussien étudiant») et pour l'un, lui attribuer un nom (Kuhn), c'est leur donner une identité, un visage, et par là, rendre à l'ennemi son côté humain. De plus, le fait de connaître le nom ou la raison sociale de ces hommes marque implicitement qu'un dialogue a été engagé entre les deux parties.

Puis, le fait de les enterrer montre un certain respect du code humanitaire, ou plutôt un retour au code de la part des Français. Cette précision s'oppose directement aux images des corps abandonnés sur les champs de bataille, sans droit aux sépultures ni à la mémoire. Les écrits rapportant cette vision particulièrement horrible de cadavres enchevêtrés et abandonnés sont malheureusement courants (Ellis 1976 : 59). L'ouvrage de Frédéric Rousseau a l'intérêt de se pencher sur la façon dont l'abandon de ces corps abandonnés est perçu par les combattants :

Le lieu normal, humain dirions-nous, de sépulture, est le cimetière. L'absence de sépulture constitue incontestablement un manquement aux rites funéraires qui choque la conscience des soldats. L'entorse aux règles semble universellement regrettée. Elle est vécue comme un manque de respect dû aux morts ; présents et futurs, car d'une certaine manière, la dévalorisation de la mort dévalorise aussi la vie. On retrouve ici l'exigence de dignité déjà perçue à plusieurs reprises. Plusieurs témoins attestent que l'abandon des morts heurte particulièrement les vivants (Rousseau 1999 : 229).

Et, s'appuyant sur plusieurs publications ${ }^{8}$, il précise que ce sentiment est ressenti différemment lorsqu'il s'agit de cadavres ennemis, sentiment d'ailleurs perceptible dans l'emploi de l'expression « un des nôtres » :

Le fait qu'il identifie ce cadavre comme « un des nôtres » est tout à fait significatif. Le combattant est généralement beaucoup moins sensible aux cadavres portant l'uniforme ennemi. [...] L'ennemi n'est pas reconnu comme un homme : alors il peut bien crever et pourrir comme un chien (ibid. : 230). 
Le contraste entre la relation aux cadavres des ennemis par les combattants français rapportée par Rousseau et l'attitude de Bénard envers les soldats allemands qu'il enterre dignement, marque nettement qu'une autre représentation de l'ennemi s'instaure. Et ce phénomène est d'autant plus singulier qu'il se produit dans le secteur de Verdun en 1916. Parce que si les modalités de la mort sont identiques sur tous les fronts de 14-18, les secteurs, quant à eux, sont différents, et Verdun est, en février, particulièrement « agité »...

Par ailleurs, signaler que le jeune étudiant "parlait bien français ", c'est le rapprocher de la France - et de l'armée française par la même occasion -, le point culturel commun étant la langue. Le partage de la même langue autorise une communication et un dialogue entre les deux nations que l'épistolier encourage et recommande. Les détails qu'il donne à sa jeune destinataire sont une façon indirecte de continuer à condamner l'absurdité de la guerre. Mais Bénard ne peut le faire littéralement (on a vu pourquoi). Le parallèle qu'il établit entre le jeune Allemand qui parlait bien français et sa fille qui apprend l'allemand n'est certes pas anodin ni innocent; la similitude invoquée dans l'étude de la langue contribue à unir la France et l'Allemagne, à déplorer la guerre et à renforcer ainsi l'effet d'absurdité.

Enfin, si le voyage prévu en Allemagne avec sa fille qu'il lui présente comme un projet cher, est un indice supplémentaire pour l'analyse, il marque également et au-delà d'une certaine camaraderie de fortune, qu'il n'y a pas non plus de haine de la part de l'épistolier vis-à-vis des Allemands.

Le rapprochement culturel élaboré dans l'échange qui s'associe à un retour au monde " civilisé » avec des ennemis qui portent un nom, auxquels sont attribués des visages et est accordée une sépulture, et avec lesquels on dialogue, sont des indices discursifs qui marquent que les données initiales du conflit (dont l'image du «barbare » exemplifiée précédemment) ont changé, ainsi que les hommes et leurs perceptions des événements. Le changement est d'autant plus sensible que Bénard présente, paradoxalement, la guerre à sa fille comme un cas forcé : "Que ce sera bon d'être sorti de cette vie que menons ", alors qu'il s'est engagé comme volontaire en 1914. C'est donc, non seulement ce qu'il élabore dans son discours qui autorise la perception du changement, mais aussi sa propre attitude par rapport à ce changement.

Seuls les échanges permettent de relever ce changement parce qu'ils étaient d'ordre privés et comme tels, s'adressaient à des proches censés comprendre ce que la censure ne pouvait percevoir par manque de données sur les correspondants. Dans le cas de Bénard, par exemple, et même en supposant que la censure sache qu'il s'agit d'un militaire de carrière retraité volontaire au combat, elle ignore que son épouse est Alsacienne mais, surtout, que son discours ne reflète plus l'homme du début de la guerre. Ce que l'analyste peut, lui, déceler dans la perspective comparative qu'offre une correspondance entière.

\section{J'ai parlé à « Fritz »}

Le jeune intellectuel Jean Pottecher, àgé de 20 ans à peine, qui s'est porté volontaire comme infirmier, arrive dans le secteur et les tranchées de Verdun. Sa correspondance comprend 350 lettres, débute le 3 septembre 1914, et comme ses camarades, le suit pas à pas et jour par jour jusqu'au bout du chemin où la mort l'attend le 24 juillet 1918 alors qu'il tente de sauver un blessé. Ses lettres sont généralement adressées à ses parents et 
plus particulièrement, à son père. Maurice Pottecher (le père de Jean), écrivain et pacifiste, fut le fondateur du théâtre du peuple de Bussang (en Lorraine) et son directeur de 1895 à 1935. Son statut, en tant que destinataire privilégié du jeune soldat, autorise des échanges fondés sur une doxa commune comme celui-ci :

Si la censure ouvre cette lettre, j'aurai évidemment des ennuis : je viens de faire une chose innocente et pourtant énorme, et qui me laisse comme au sortir d'un rêve : j'ai parlé à Fritz. Une section de la $7^{\mathrm{e}}$, juste devant, est à 20 mètres environ des sentinelles boches; ils échangent des promesses : «Vous pas tirer, pas tirer ». Je suis allé les voir. [...] J'avais affaire à un sous-officier boche, et j'étais à côté d'un sous-lieutenant français, ancien adjudant rengagé, et qui m'arrêtait à chaque instant : si bien que j'ai été gêné et que la confiance n'a pas régné. On voyait la tête de deux Boches en calot, en assez bonne forme, mais sales comme moi, l'un tout jeune, l'autre un sous-officier, de 25 à 30 ans. Ils regardaient le sous-lieutenant français et le célèbre Garrier, qui leur faisait signe de boire. J'arrive et je crie: «Bonjour! Cela va bien" ? ». Ils n'entendent pas, et je répète : «Cela va bien ${ }^{10}$ ? » Ils me répondent tous deux : "Non, cela ne va pas bien ${ }^{11}$ " et montrent leur boue. Je leur demande: "Avez-vous à boire et à manger ${ }^{12}$ ? IIs répondent d'un geste indifférent, et me font signe de venir : «Vous venir. Venez ${ }^{13}$ ! ». «Impossible ${ }^{14}$. » Ils insistent beaucoup. Je demande : «Quand la paix viendra-t-elle ${ }^{15}$ ? » C'est là qu'ils m'ont épaté par un nouveau geste indifférent. Sans me gêner, je continue: " espérons tous que ce sera bientôt ». «Oui ${ }^{16}$ ». Je montre mon brassard. «Vous êtes le médecin ${ }^{17}$ ? " me demandent-ils. « oui ${ }^{18}$ ! (pour éviter trop d'explications : je ne sais pas dire « infirmier»). À la demande du sous-lieutenant, je m'en vais. [...] et je reviens au poste élevé. Je les rappelle: «D'où êtes-vous? De quelle partie de l'Allemagne venez-vous ${ }^{19}$ ». Je n'ai pas compris la réponse. Je demande : « N’y a-t-il parmi vous personne de la Bavière, de Munich. J'ai passé tout un mois à Munich ${ }^{20}$.» « Non, personne ${ }^{21}$. " Je répète : « Êtes-vous de la Saxe ? Du Schleswig ? De la Prusse ${ }^{22}$ ?... » Ils répondent : "On ne doit pas le dire ${ }^{23}$. " Le sous-lieutenant me pressant, je leur dis : "ça ne fait rien. Au revoir. Adieu ${ }^{24}$ !» Ils me saluent d'un geste amical. Et voilà tout! Quel mal à s'entendre, et quel bien ce serait! (Pottecher [1926] 2003 : 77,78 ; lettre à ses parents du 17 décembre 1916).

Précisons avant toute chose que le texte original des dialogues que rapporte Jean est en langue allemande $\mathrm{e}^{25}$, langue connue des interlocuteurs par leur origine géographique et culturelle commune qu'est la Lorraine, annexée par l'Allemagne après 1870. Dans l'échange, Pottecher "fait plusieurs choses». Tout d'abord, il introduit d'emblée son désir de fraternité avec les Allemands en présentant le dialogue instauré entre les deux armées comme " une chose innocente » et irréelle « qui me laisse comme au sortir d'un rêve ». L'emploi du terme "rêve", du registre de la fiction et synonyme de souhait, d'aspiration, voire, dans certains cas, d'idéal, marque que pour Pottecher, la situation lui apparait "invraisemblable » (comme pour Bouchet) et révèle en même temps sa parfaite connaissance de l'interdit qu'il enfreint consciemment. Il adopte ainsi une attitude épistolaire qui consiste à rompre avec les règles jusqu'ici respectées (parler avec l'ennemi) et entend la «montrer» discursivement en anticipant sur les conséquences qu'il affiche dans son discours comme une responsabilité assumée. Cette attitude marque en même temps une certaine désinvolture par rapport aux graves sanctions qu'il encourt: "Si la censure ouvre cette lettre, j'aurai évidemment des ennuis " (qu'il minimise par l'emploi du terme "ennuis ») Il pose donc son choix de communiquer avec l'ennemi comme un acte délibéré. La délibération personnelle qu'il affiche ici est directement liée au statut de ses destinataires dont son père principalement (le socialiste Maurice Pottecher), auquel il accorde un rang privilégié de confident et dont l'opinion à ses yeux présente une valeur certaine. 
Notons que précédemment, Bouchet et Bénard avaient déjà marqué cette attitude de détachement par rapport aux règles, mais d'une façon différente. Bouchet avait choisi l'implicite et s'était servi de la comparaison dans les difficiles conditions des tranchées. Quant à Bénard, il avait plutôt opté pour une "humanisation » de l'ennemi à travers des portraits et des descriptions, et le point culturel qu'est la langue allemande.

Ensuite, l'épistolier opère également et comme Bouchet, une identification entre les deux armées dans les pénibles conditions qu'ils partagent "sales comme moi, ils montrent leur boue». La comparaison introduite par «comme» justifie le rapprochement des hommes dans une expérience commune. Ici, la saleté et la boue, lot commun des deux armées, est une raison suffisante, aux yeux de l'épistolier, pour porter une évaluation négative sur leurs conditions lamentables de combat (et de vécu) qui justifie sa décision de "dialoguer ». Ajoutons à cela que la boue et la saleté ne sont que des facteurs supplémentaires, des arguments qui s'ajoutent à beaucoup d'autres, plus graves, accumulés au fil du conflit, tels que les carnages, la faim, le froid, etc. Cette terrible situation que Pottecher attribue aussi bien aux Français qu'aux Allemands explique donc sa perception de «l'ennemi » dont il élabore (comme Bénard) un portrait humain : il leur donne un âge, des fonctions «l'un tout jeune, l'autre un sous-officier, de 25 à 30 ans ".

Enfin, dans l'expression «Quel mal à s'entendre, et quel bien ce serait! », l'emploi du conditionnel a pour fonction de souligner l'improbabilité du souhait et en même temps le mode irréel qui rappelle le « rêve » et « l'innocence » du début du passage. Cette mise en mots consiste à « attribuer au monde réel des caractères irréels » (Ducrot 1973 : 110). Elle montre que Pottecher est parfaitement conscient du caractère irréel de ce qu'il avance tout en le présentant comme souhaitable, et marque nettement un changement de représentations par rapport aux discours officiels antérieurs qui charriaient la haine de l'ennemi. Il rejoint d'ailleurs le discours de Bénard «Que ce sera bon d'être sorti de cette vie que menons ! » flanqué d'un point d'exclamation. Ce dernier, qui accompagne généralement les interjections, a une valeur affective et fait passer l'expression d'une émotion. Il est « le lieu où se marque l'interaction des individus» (Ducrot $1980: 161$ ). Dans le cas de Pottecher, Bénard et Bouchet, nous l'aurons compris, il s'agit de faire comprendre à leurs destinataires respectifs que l'ennemi est perçu différemment et que leurs désirs se résument à l'arrêt des tirs (même provisoire) et à une certaine « humanisation » des hommes, quel que soit l'uniforme qu'ils portent. Ce dernier point marque, par ailleurs, une lassitude générale vis-à-vis des carnages particulièrement nombreux à cette époque de la guerre et en ce lieu.

On voit que chaque épistolier utilise son bagage individuel, qu'il soit d'ordre culturel, social ou encore politique, et celui commun avec son destinataire pour lui faire comprendre qu'un changement repérable au niveau de la perception et de la représentation discursive de l'ennemi est en train de s'opérer, mais aussi qu'un changement beaucoup plus vaste qui conditionne cette attitude, est en cours.

\section{Conclusion}

Les exemples sont encore nombreux, quelle que soit la correspondance que l'on explore à cette période de combats incessants et meurtriers (entre janvier et décembre 1916). L'échantillon des épistoliers présentés, volontairement hétérogène afin de respecter la diversité mais aussi la particularité de chacun, montre que malgré ces critères, les 
combattants semblent se rallier à une voix collective qui aspire à une "trêve » des combats, du jeune artilleur rural à l'officier de carrière en passant par le jeune intellectuel. Pourtant, et aussi paradoxal que cela puisse paraître, étant donné le secteur et l'époque, le discours épistolaire de cette année livre un grand nombre d'échanges qui "parlent » implicitement ou explicitement de "trêve ». S'il est exact que quelques rares épistoliers s'étaient risqués à en parler dans leurs échanges dès 1914, comme Hertz ${ }^{26}$, par exemple, ou encore en 1915, comme Barbusse ${ }^{27}$, l'année 1916 est beaucoup plus riche en exemples et les discours plus explicites sur le sujet. Par ailleurs, l'appellation de ces " évènements d'accalmie " reste très variable en fonction des circonstances, de ceux qui l'emploient et des perceptions de chacun. Bouchet parle d'« entente cordiale », Pottecher s'interroge "Quel mal à s'entendre » et Bénard aspire à « sortir de cette vie que menons ». D'autres encore, comme l'historien Jules Isaac, font allusion à une "convention tacite»: "Mais, par une convention tacite, on feint de s'ignorer, chacun étant suffisamment occupé à pomper pour atténuer dans la mesure du possible le bain de pieds obligatoire » (Isaac 2004 : 184 ; lettre du 16 avril 1916 à son épouse). Pour l'institution militaire, ces "activités » se résument en un seul et même terme : fraternisation. Les soldats, eux, ne l'utilisent jamais littéralement. Tout au plus le suggèrent-ils par la construction discursive de l'image du combattant allemand, par des comparaisons établies entre les deux armées, ou encore par l'utilisation de prédicats.

Si les stratégies discursives sont variées, leur finalité est identique : elles marquent un changement dans la représentation de l'ennemi et impliquent une modification dans l'échelle des valeurs. Cette dernière est la conséquence d'un vécu sur le terrain que les destinataires de l'arrière ne peuvent connaître ni, peut-être même, comprendre. Pour cette raison, les combattants mettent en place une argumentation susceptible de les faire adhérer à leur nouvelle vision de l'ennemi, malgré la "rupture " par rapport à une doxa partagée qui fait peser une menace sur leur ethos.

On l'aura remarqué, l'échantillon étudié marque un rapport nuancé sur la question de l'image de l'ennemi. Pour cette raison, il me semble pertinent de se référer pour les " cas d'accalmie » mis en mots dans l'étude, à la formule proposée dans les travaux de l'historien anglais Tony Ashworth à propos du front britannique «vivre et laisser vivre " (Trench Warfare. Live and let Live System, 1980). L'auteur se réfère à des accalmies du front, des «ententes tacites » qui permettent aux combattants des deux armées de se ravitailler en eau, nourriture et de s'affairer à d'autres tâches quotidiennes vitales. C'est dans ce sens que je conçois le terme de "fraternisation", dans le sens où les combattants autorisent ces occupations sans tirer les uns sur les autres. Cette attitude se construit et se "dit» dans les discours des combattants en s'imposant comme un contraste par rapport à un vécu précédent. En effet, les analyses des correspondances qui sont submergées de termes tels que marmitage, carnage, implacable enfer, fouillis (de corps humains), immortel calvaire, et d'images d'innombrables cadavres, laissent percevoir que, peu à peu, ce lexique fait place à un autre, différent, qui traduit une nette accalmie. C'est ce glissement lexical, ce contraste entre des tirs incessants relatés dans les lettres sur "tout ce qui bouge" (et leur conséquence), et le calme qui s'instaure par la suite à ce sujet dans les échanges, qui a fait surgir le terme naturellement. Ce changement d'attitude (ne pas tirer pour " vivre et laisser vivre ») est perceptible dans le contexte de longues correspondances suivies et émerge progressivement. D'ailleurs, rappelons-le, les épistoliers ont eux-mêmes attribué des dénominations différents à cette situation : certains ont parlé « d'entente cordiale » 
(Bouchet) ou encore de «convention tacite» (Isaac) comme si le terme « fraternisation » était déjà trop lourd de significations pour eux aussi...

La mise en mots constatée dans les échanges analysés autorise l'élaboration d'une nouvelle représentation de l'ennemi mais aussi, et, en même temps, une nouvelle représentation des gouvernants et de l'institution militaire. A l'opposition Français/ Allemands vient se substituer le rapport des combattants des deux bords unis par de similaires souffrances et privations. Cette nouvelle perception de «l'ennemi » en entraînera inévitablement une autre inhérente aux gouvernants civils et aux chefs militaires de plus en plus critiqués, et remettra en cause la question de la discipline et du respect hiérarchique. Mais ce dernier point, très complexe, mérite de faire l'objet d'une autre analyse.

\section{BIBLIOGRAPHY}

Amossy, Ruth. 2010 [2000]. L'argumentation dans le discours (Paris : Colin)

Ashworth, Tony. 1980. Trench Warfare 1914-1918: The Live and Let Live System (New-York: Holmes \& Meier)

Audoin-Rouzeau, Stéphane \& Jean-Jacques Becker. 1995. La France, la nation, la guerre : 1850-1920 (Paris : Sedes)

Audoin-Rouzeau Stéphane \& Annette Becker. 2000. 14-18 retrouver la guerre (Paris : Gallimard) Barbusse, Henri. 1937. Lettres de Henri Barbusse à sa femme 1914-1917 (Paris : Flammarion)

Barthas, Louis [1977] 2003. Les carnets de guerre de Louis Barthas, tonnelier, 1914-1918 (Paris : La Découverte)

Becker, Jean-Jacques. 2004. La Grande Guerre (Paris : PUF)

Bénard, Henri. 1999. De la mort, de la boue, du sang : Lettres de guerre d'un fantassin de 14-18 (Paris : Grancher)

Bouchet, Raoul. 2002. Lettres de guerre d'un artilleur de 1914 à 1916 (Paris : L'Harmattan)

Brown Malcom, Rémy Cazals \& Olaf Mueller. 2005. Frères de tranchées, Ferro Marc (dir.) (Paris : Perrin)

Cazals, Rémy \& Frédéric Rousseau. 2001. 14-18 le cri d’une génération (Toulouse : Privat)

Delvau, Alfred. 1883 [1866]. Dictionnaire de la langue verte. Argots parisiens comparés (Paris : Marpon \& Flammarion)

Ducrot, Oswald. 1973. La preuve et le dire (Paris : Mame)

Ducrot, Oswald. 1980. Les mots du discours (Paris : Minuit)

Ducrot, Oswald. 1984. Le dire et le dit (Paris : Minuit)

Ellis, John. 1976. Eye-Deep in Hell, Trench Warfare in World War 1 (Baltimore: Johns Hopkins University Press) 
Gazin \& Segonne. 1931. Cours d'Histoire Guerre 1914-1918, Lithographie de l'École militaire et d'application du Génie, D.C - E.O.R ; Approbation Ministérielle No 324 3/4 du 30 Janvier 1931

Goffman, Erving. 1973. La mise en scène de la vie quotidienne (Paris : Minuit)

Hertz, Robert. 2002. Un ethnologue dans les tranchées (Paris : CNRS Éditions)

Horne, John (dir.). 2010. Vers la guerre totale. Le tournant de 1914-1915 (Paris : Tallandier)

Horne John \& Kramer Alan. [2001] 2005. German Atrocities, 1914. A History of Denial (New Haven \& Londres : Yale University Press (Éditions Tallandier 2005, pour la version française).

Isaac, Jules. 2004. Un historien dans la Grande Guerre (Sejer : Colin)

Jakobson, Roman. 2003 [1963]. Essais de linguistique générale, 1. Les fondations du langage (Paris :

Minuit)

Kerbrat-Orecchioni, Catherine. 1978. " Déambulation en territoire aléthique ", Stratégies

discursives, Actes du colloque du Centre de Recherches linguistiques et sémiotiques de Lyon, 20-22 mai 1977 (Lyon : PUL), 60-61

Kerbrat-Orecchioni, Catherine. 1986. L'implicite (Paris: Colin)

Papillon. 2003. Si je reviens comme je l'espère Postface de Rémy Cazals \& Nicolas Offenstadt (Paris : Bernard Grasset)

Perelman, Chaïm. 2002. L'empire rhétorique (Paris : Vrin)

Pottecher, Jean. 2003 [1926]. Lettres d'un fils, un infirmier de chasseurs à pied à Verdun et dans l'Aisne (Louviers : Ysec)

Prost, Antoine \& Jay Winter. 2004. Penser la Grande Guerre (Paris : Seuil)

Rousseau, Frédéric. 1999. La guerre censurée (Paris : Seuil)

Sainean, Lazare. 1973 [1915]. L'argot des tranchées, d'après les lettres des poilus et les journaux du front (Genève : Slatkine)

Strachan, Hew. 2005 [2003]. La première guerre mondiale (Paris : Presses de la Cité)

\section{NOTES}

1. Pour plus de renseignements sur la question de «l'ennemi", voir Horne et Kramer 2005 [2001].

2. Sur ce sujet, voir Brown, Cazals et Mueller (2005).

3. Comme par exemple Galilipoli et Salonique

4. Dans le sens d'être au mauvais endroit, au mauvais moment

5. On donnera pour exemple, l'image choisie par L'Illustration du 20 août 1914, no 3731, qui montre un soldat allemand, fusil à la main, debout et souriant le pied sur le corps d'une femme inanimée au sol, entourée d'un enfant, d'un prêtre et d'autres civils eux aussi gisant. La photo qui rappelle celle du chasseur avec son trophée, est intitulée «leur façon de faire la guerre » et le commentaire précise, entre autres : « Il y a eu autrefois des hordes humaines qui l'ont faite ainsi, et ne concevaient point, en leurs cervelles de brutes sauvages que la conquête, ou simplement la guerre, ce pût être autre chose que la destruction et l'extermination de tout [...] Ou doit-on croire qu'au fond de toutes ces âmes germaines dormait le vieil instinct de barbarie... ». 
6. Le soldat fait plutôt son devoir et obéit aux ordres, comme le montre bien Bouchet: «Les Russes continuent à faire du bon travail et attendons que l'ordre d'en faire autant ... » (2002: 286, 21 juin 1916).

7. A la fois bourgade à $20 \mathrm{kms}$ au sud-est de Verdun et crête qui forme la ligne d'arête des Hauts de Meuse. Excellent observatoire naturel dominant la plaine de la Woëvre.

8. Evelyne Desbois, article Grand Guignol. Blessés et mutilés de la Grande Guerre paru en mars 1992 dans Terrain no. 18 ; Edgar Morin, L'Homme et la mort paru en 1951 (Seuil) ; Ma pièce, journal de guerre de Paul Lintier, artilleur de 14-18 et mort en mars 1916 (paru en mai 1916).

9. Guten Tag! Geht's gut?

10. Geht's gut?

11. Nein, s'ist nicht gut

12. Ihr habt Wasser und Kot [sic, à la place de « Brot »]?

13. Komm!

14. Unmoeglich! (réponse de Pottecher aux soldats allemands)

15. Wann wird es Friede?

16. Ja (réponse des soldats allemands à Pottecher)

17. Sie sind der Altlz?

18. Ja!

19. Woher seid ihr? Von welcher Deutschland's Teil kommt ihr her?

20. Giebt es keiner von Bayern, von Munchen? Ich habe einen Monat lang zu Munchen zugebracht

21. Nein, es giebt keiner [sic - réponse des Allemands à Pottecher]

22. Seid ihr von Sachsen? Schlesien? Preussen?

23. Das darf man nicht sagen

24. S'macht nichts! Auf Wiedersehen! Ade !

25. Précisons que le texte original figurant en note a été transcrit tel quel (avec les fautes d'allemand).

26. Voir sa très longue lettre du 25 décembre 1914 à son épouse (Hertz 2002 : 163)

27. "Sur certaines parties de notre front, l'impossibilité de se maintenir dans les tranchées de première ligne, telles qu'elles sont, et la nécessité de les réparer ont amené Français et Allemands à se montrer à découvert sans se tirer de coups de fusil » (Barbusse 1937 : 188. Lettre à son épouse du 11 décembre 1915).

\section{ABSTRACTS}

This paper explores the epistolary discourse of French soldiers during WWI, and more specifically the letters written from the battlefield of Verdun in 1916. An in-depth analysis of linguistic, cultural and historical elements shows how these private letters endeavored not only to communicate points of views, perceptions and personal emotions, but also justify them in order to elicit some kind of adhesion from the addressee. Examining the rhetoric of the war letter will thus throw light on the way the fighters experienced and understood the conflict, and tried to account for their feelings and behavior. In this framework, we examine how the private discourse of the combatants fighting on the battlefield of Verdun constructs, in the epistolary exchange, an unexpected image of the enemy, namely, the German soldier. Also, the paper shows 
how certain representations originally circulated by the media and official discourse are reelaborated and modified, so that identification can occur between the two camps who participate in the same suffering and destiny. Such an analysis of epistolary discourse allows for a better understanding of the phenomenon of "fraternization" that is at the center of a polemical debate between contemporary historians of WW1.

L'article explore le discours épistolaire des combattants français durant la Grande Guerre, et plus particulièrement les lettres rédigées sur le front de Verdun en 1916. Il montre comment, à travers une analyse ponctuelle d'éléments langagiers mais aussi culturels et historiques, ces lettres privées ont servi non seulement à communiquer des points de vue, des perceptions et des émotions personnelles, mais aussi à les justifier afin d'obtenir une certaine adhésion de la part des destinataires. L'analyse des représentations discursives permet d'effectuer une réflexion sur la mise en mots à partir de laquelle s'établit le sens profond du conflit pour les soldats des tranchées, et d'éclairer les raisons qui conditionnent leurs actes et leurs façons de penser. Dans cette perspective, on voit comment le discours privé des combattants du front de Verdun construit dans l'échange une image pour le moins surprenante et inattendue de l'ennemi, en l'occurrence du soldat allemand. On montre, par ailleurs, comment certaines représentations initialement charriées par les médias et les discours de propagande sont retravaillées et déplacées, et comment une identification entre les deux camps dans une souffrance et un destin communs mène à une justification, parfois implicite, du très combattu " rapprochement » entre les deux armées, même au cœur des combats. Une analyse approfondie du discours épistolaire permettra ainsi une meilleure compréhension du phénomène "fraternisation », dans le débat des historiens sur la Grande Guerre.

\section{INDEX}

Mots-clés: analyse du discours, argumentation, combattants français, discours épistolaire, Grande Guerre, image de l'ennemi, trêves des combats

Keywords: argumentation, discourse analysis, epistolary discourse, French soldiers, image of the enemy, truces in fighting, WW1

\section{AUTHOR}

\section{SYLVIE HOUSIEL}

Université de Tel-Aviv, ADARR 